\title{
Comparison of videolaryngoscopy and direct laryngoscopy by paramedics during out of hospital CPR An observational prospective study
}

Joachim Risse ${ }^{1}$, Christian Volberg², Thomas Kratz ${ }^{3}$, Birgit Plöger ${ }^{2}$, Andreas Jerrentrup ${ }^{2}$, Clemens Kill ${ }^{1}$

1 Universitätsmedizin, Essen, Germany. 2 Universitätsklinikum, Marburg, Germany. 3 SELARL d'Anesthésie Réanimation, Clinique Bénigne Joly, Talant, Côte-d'Or, France

Center of Emergency Medicine, University Hospital Essen, Germany

\section{Purpose of the study:}

Although videolaryngoscopy (VL) may facilitate intubation in rescuers with low experience there is limited data available concerning endotracheal intubation performed by paramedics during out of hospital CPR. The goal of this trial was to investigate endotracheal intubation during CPR performed by non-physicians in a single emergency department in Germany.

\section{Materials and methods:}

We investigated in an observational prospective study the efficacy of VL during CPR compared to conventional direct laryngoscopy $(\mathrm{DL})$ performed by paramedics. After approval by local ethics committee, we instructed and equipped paramedics of four ambulances in endotracheal intubation with GlideScope ${ }^{\circledR}(G V L)$. In cases without presence of an EMS physician on scene intubation should be performed either with GVL or with DL. A maximum of two attempts were allowed per protocol. Primary endpoint was good visibility of the glottis (Cormack Lehane grading1/2), secondary endpoint successful intubation

Corresponding Author: Joachim Risse, MD, Center of Emergency Medicine, University Hospital Essen, Hufelandstrasse 55 45122 Essen, Germany, email: joachim.risse@uk-essen.de

RESUSCITATION 2018 New technologies in resuscitation $20-21-22$ September $\bullet$ Bologna $\bullet$ Italy

\section{Results Part I:}

In total $n=97$ patients could be included, $n=69$ using direct laryngoscopy (with $n=85$ intubation attempts) and $n=28$ using videolaryngoscopy (with $n=37$ intubation attempts). Laryngoscopy with GVL resulted in a significantly improved visualization of the larynx compared to $\mathrm{DL}$. In the group using GVL $82 \%$ rated visualization of the glottis as $C L 1 \& 2$ versus $55 \%$ in the $\mathrm{DL}$ group $(p=0.02)$. Despite better visualization of the larynx there was no statistical significant difference in successful endotracheal intubation between videolaryngoscopy and direct laryngoscopy (GVL $75 \%$ vs. DL $68 \%, p=0.63$ ). In addition, more attempts for the successful endotracheal intubation were needed in the GVL group.

Table 1. Comparison View of the Larynx by Cormack-Lehane Classification System: Videolaryngoscopy (GVL) vs. Direct Laryngoscopy (DL)

\begin{tabular}{llll}
\hline $\begin{array}{l}\text { Dichotomized } \\
\text { CL-Grade }\end{array}$ & GVL & DL & P-value \\
\hline I+II (easier ETI) & $23(82.15 \%)$ & $38(55.7 \%)$ & $\mathbf{0 . 0 2}$ \\
III+IV (difficult ETI) & $5(17.85 \%)$ & $31(44.93 \%)$ & $\mathbf{0 . 0 2}$
\end{tabular}

\begin{tabular}{llll}
\hline CL-Grade & GVL & DL & P-value \\
\hline I & $11(39.29 \%)$ & $8(11.59 \%)$ & $\mathbf{0 . 0 0 4}$ \\
II & $12(42.86 \%)$ & $30(43.48 \%)$ & 1 \\
III & $3(10.71 \%)$ & $20(28.99 \%)$ & 0.07 \\
IV & $2(7.14 \%)$ & $11(15.94 \%)$ & 0.34 \\
\hline
\end{tabular}

Results Part II:

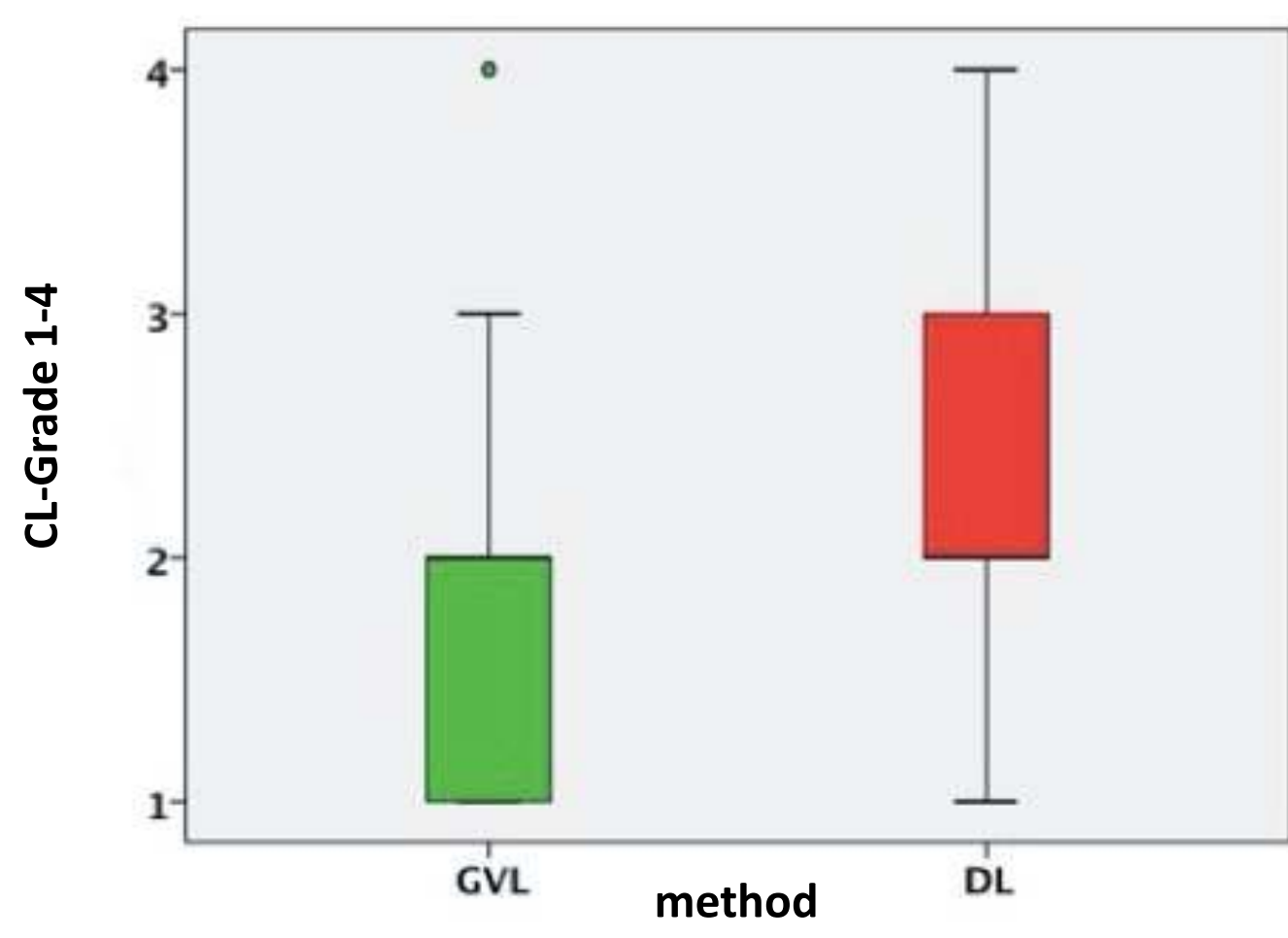

Table 2. Comparison of Overall Successful Endotracheal Intubation (ETI)

\begin{tabular}{c|cc|c}
\hline \multicolumn{5}{|c}{ ETI successful } & ETl unsuccessful \\
\hline GVL & $21(75 \%)$ & $7(25 \%)$ & 28 \\
DL & $47(68.1 \%)$ & $22(31.9 \%)$ & 69 \\
\cline { 2 - 4 } & 68 & 29 & 97 \\
\hline & \multicolumn{2}{|c|}{ Fisher's exact test two-tailed P-value is 0.626777} \\
\hline
\end{tabular}

Conclusions:

When used by paramedics during CPR GVL leads to better visibility of the glottis, but did not increase the number of successful initial intubation attempts.

Therefore, we conclude that education in videolaryngoscopy should also focus on the insertion of the endotracheal tube. 